Chronic Obstructive Pulmonary Diseases:

Journal of the COPD Foundation

\author{
Original Research
}

\title{
Validation and Assessment of the COPD Treatment Ratio as a Predictor of Severe Exacerbations
}

Richard H. Stanford, PharmD, $\mathrm{MS}^{1,2}$ Stephanie Korrer, $\mathrm{MPH}^{3}$ Lee Brekke, $\mathrm{PhD}^{3}$ Tyler Reinsch, $\mathrm{PharmD}^{1}$ Lindsay G.S. Bengtson, $\mathrm{PhD}^{3}$

\section{Abstract}

Background: Population-based risk assessments are needed to identify individuals who may benefit from chronic obstructive pulmonary disease (COPD) management programs for preventing exacerbations. This study compared the validated COPD treatment ratio (CTR) versus other COPD exacerbation predictors: prior exacerbation and rescue and maintenance medication use.

Methods: A retrospective observational study using medical and pharmacy claims data among Medicare Advantage with Part D beneficiaries with COPD (January 2011-August 2016). Unadjusted and adjusted logistic regression models tested the predictive performance ( $C$-statistic) of potential exacerbation predictors for future severe exacerbations.

Results: The unadjusted association between exacerbation predictors and severe exacerbation was examined in 60,776 patients: baseline severe exacerbation had the highest $C$-statistic (0.668), then number of rescue units dispensed (0.651), CTR (0.619), and number of controller units dispensed (0.562). During the at-risk period, baseline CTR was inversely associated with severe exacerbation (odds ratio, <1.0); other predictors were positively associated with a severe exacerbation (odds ratio, >1.0). Adjusting for age, geographic region, chronic oxygen, and nebulizer use, the severe exacerbation odds were 0.90 (95\% confidence interval [CI], 0.89-0.91) lower per 0.10 change in CTR ( $C$-statistic, 0.710$)$. The $C$-statistic was 0.734 when baseline exacerbation was added to the model.

Conclusions: The CTR is an effective tool for identifying patients diagnosed with COPD who are at increased risk of severe exacerbation. Although CTR does not predict future exacerbation as well as prior severe exacerbation history, it has the advantage of being applicable in predicting future exacerbations in patients without an exacerbation history, or in databases limited to pharmacy claims only. In addition, the significant reduction in risk has been observed with incremental increases in the ratio: the ratio can be monitored to assess COPD health improvements over time.

Abbreviations: chronic obstructive pulmonary disease, COPD; COPD treatment ratio, CTR; confidence interval, CI; Body mass index, airflow Obstruction, Dyspnea, and Exercise, BODE; emergency department, ED; Medicare Advantage with Part D coverage, MAPD; International Classification of Diseases, $9^{\text {th }}$ Revision, Clinical Modifications, ICD-9-CM; odds ratio, OR; asthma medication ratio, AMR

Funding Support: Sponsored and funded by GlaxoSmithKline plc (HO-16-17938).

Date of Acceptance: December 19, 2019

Citation: Stanford RH, Korrer S, Brekke L, Reinsch T, Bengston LGS. Validation and assessment of the COPD treatment ratio as a predictor of severe exacerbations. Chronic Obstr Pulm Dis. 2020;7(1):38-48. doi: https://doi.org/10.15326/jcopdf.7.1.2019.0132

1 Strategic Consulting, AESARA, Inc., Chapel Hill, North Carolina

2 Department of Pharmaceutical Outcomes and Policy, University of North Carolina, Chapel Hill

3 Health Economics and Outcomes Research, Optum Inc., Eden Prairie, Minnesota

\section{Keywords:}

chronic obstructive pulmonary disease; COPD; COPD treatment ratio; CTR; COPD exacerbation; maintenance medication; rescue inhaler 


\section{Address correspondence to:}

Richard H. Stanford, PharmD, MS

Strategic Consulting

AESARA, Inc.

Chapel Hill, NC

Email: richard@aesara.com

Phone: (919) 6020283

\section{This article has an online data supplement.}

\section{Introduction}

Chronic obstructive pulmonary disease (COPD) is a progressive, treatable disease of the airways associated with substantial morbidity and mortality. ${ }^{1}$ In the United States, COPD was the third leading cause of death in 2015 , with an estimated age-adjusted prevalence of $5.9 \%{ }^{2}$ The medical costs attributable to COPD were approximately $\$ 36$ billion in 2010 and are projected to reach $\$ 49$ billion by $2020 .^{3}$

Exacerbations are experienced by patients with COPD at all stages of disease severity. ${ }^{4}$ Hospital visits associated with acute exacerbations are linked to increased morbidity, increased mortality, and reduced quality of life, ${ }^{5}$ and account for $85 \%$ of direct medical costs due to COPD.,7 To improve patient outcomes and reduce health care utilization and costs, population-based risk assessment tools are needed to identify patients who may benefit from interventions that prevent COPD exacerbations. ${ }^{8}$ Therefore, developing valid predictors of COPD exacerbations through a more robust understanding of associated treatment factors, such as maintenance and rescue medication use, is an important goal.

Exacerbation risk models using clinical information have been developed previously, such as that by Niewoehner et $\mathrm{al}^{9}$ and the Body-mass index-airflow Obstruction-Dyspnea-Exercise (BODE) index, ${ }^{10}$ which predicted exacerbations based on advanced age, COPD severity, unscheduled clinic or emergency department (ED) visits, cardiovascular comorbidity, and prednisone use; and body mass index, airflow obstruction, functional dyspnea, and exercise capacity, respectively. More recently, 2 further studies utilized exacerbation history, lung function, smoking, and vascular disease history in their validation dataset, ${ }^{11}$ and patient responses to the COPD Assessment Test, ${ }^{12}$ to determine exacerbation risk. In the past, risk scores used to predict acute COPD exacerbations have relied on information from medical records that were not always readily available to external researchers. ${ }^{11,12}$

The COPD treatment ratio (CTR) is a new type of risk score and modifiable measure that uses accessible prescription data within health insurance claims, that has been developed as an easy method to assess exacerbation risk, without the need for patient history data. ${ }^{13}$ It is defined as the total units (30-day equivalent) of maintenance medication dispensed, divided by total units of maintenance plus rescue medications dispensed. The CTR has been validated in previous retrospective studies using health insurance data and has the potential to identify patients with an increased exacerbation risk who may benefit from interventions to improve treatment adherence, which in turn, may allow for the development of modifiable patient management. ${ }^{13,14}$

We compared the CTR ${ }^{13,14}$ as a predictor of severe exacerbations versus other COPD exacerbation predictors available in claims data: prior exacerbation, and use of rescue and maintenance medications.

\section{Methods}

\section{Data Source}

Data were extracted from the Optum Research Database, a large, geographically diverse U.S. administrative claims database that includes medical and pharmacy claims for individuals enrolled in Medicare Advantage with Part D (MAPD) health plans.

\section{Study Design and Sample}

This retrospective, observational study used health care claims among MAPD beneficiaries diagnosed with COPD to validate CTR as a modifiable measure of COPD exacerbation risk. Medical and pharmacy data, and enrollment information from January 1, 2011 to August 31, 2016, were used (Figure 1). Eligible patients were $\geq 40$ years of age as of the year of the index date, had $\geq 1$ pharmacy claim for a COPD maintenance medication during the baseline period, continuous medical and pharmacy coverage for $\geq 2$ years following the index date, and had at least 1 inpatient hospitalization or 2 outpatient and/ or ED visits within 1 year (defined by an International Classification of Diseases, 9th Revision, Clinical Modification [ICD-9-CM] COPD diagnosis code of 491.x, 492.x, or 496 in any position [the identification period ended on August 31, 2014; therefore only 


\section{Figure 1. Study Design}

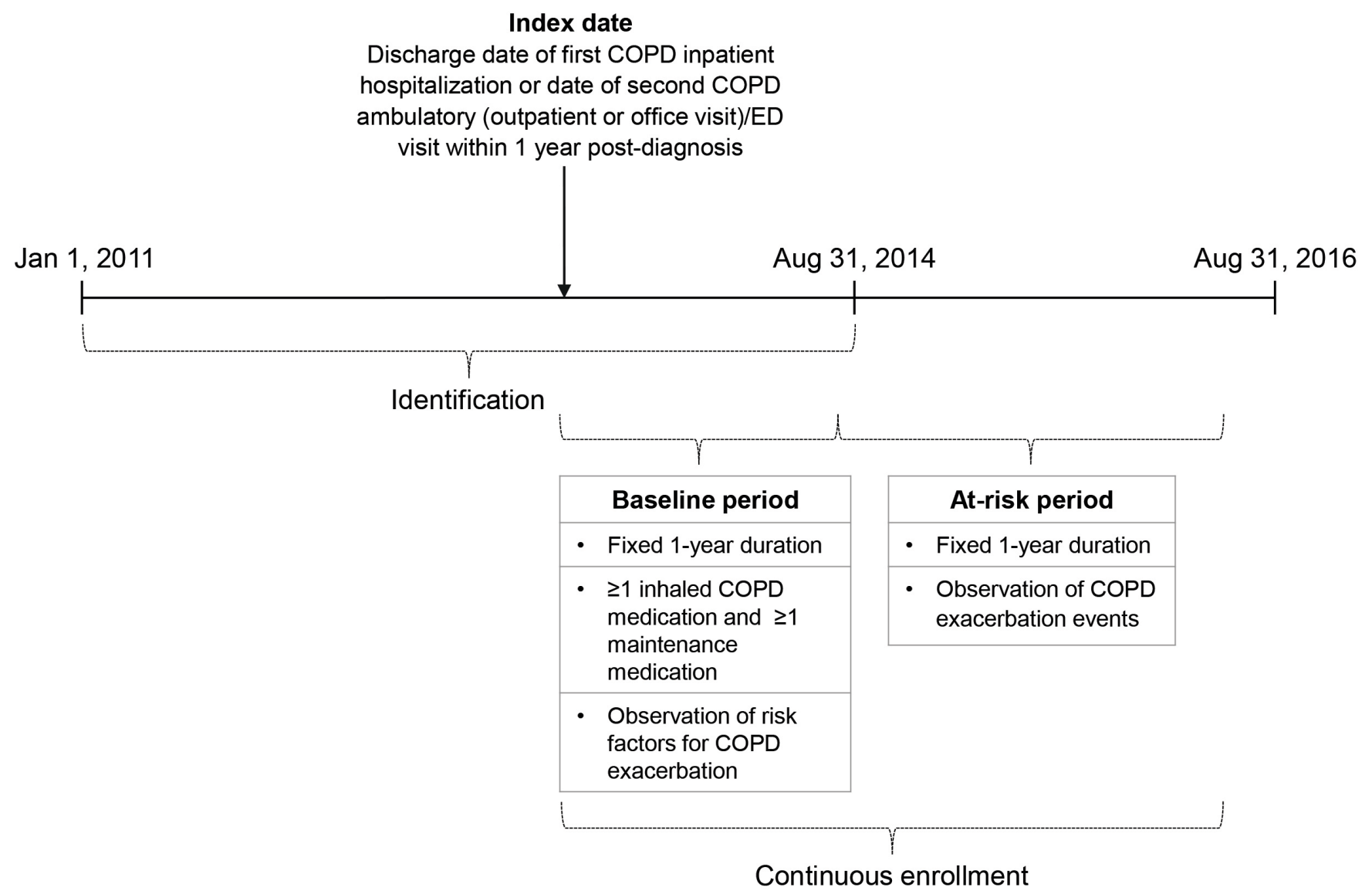

$\mathrm{COPD}=$ chronic obstructive pulmonary disease; $\mathrm{ED}=$ emergency department

ICD-9 codes and not ICD-10th Revision (ICD-10) codes were included]).The index date was defined as the earlier of either: (a) the discharge date of the first COPD inpatient hospitalization or (b) the date of the second COPD-related ambulatory (outpatient or office visit) and/or ED visit within a 1-year period; the second visit date was used to ensure the eligibility criteria had been met at the index date and to prevent confounding of the exacerbation income. The index date was used to identify a baseline period (1-year post-index) and a subsequent at-risk period (1-year post-baseline; Figure 1).

As use of maintenance medication during the baseline period was required, 24,967 patients with a CTR of 0 (no maintenance medication) were excluded. Patients diagnosed at baseline with any type of cancer were excluded, except for those with $\geq 1$ non-diagnostic claims at baseline with an ICD-9-CM diagnosis code for a cancer with typically little effect on lung function (i.e., breast, prostate, and non-melanoma skin cancers). ${ }^{13}$

Severe exacerbations were the focus of analysis due to previous evidence for the ability of the CTR to better predict severe exacerbations and more consistently predict time to first severe exacerbation. ${ }^{14}$

\section{Medication Classes Used to Calculate the COPD Treatment Ratio}

Two classes of medication were used to calculate the CTR: maintenance medication and rescue medication. Maintenance medication included longacting anti-muscarinic agents, long-acting-beta2agonists, inhaled corticosteroids, methylxanthines, and phosphodiesterase-4 enzyme inhibitor rescue. Rescue medication included short-acting beta2-agonists and short-acting anti-muscarinic agents, either alone or combined. 


\section{Predictor Variables and Covariates}

The 4 predictor variables of interest were: (1) baseline CTR (defined as the total units of maintenance medication dispensed, divided by total units of maintenance plus total units of rescue medications dispensed during the baseline period); (2) any (severe or moderate) baseline exacerbation; (3) count of baseline rescue units dispensed (1 unit of a rescue medication was defined as a canister of a rescue medication [containing 100 doses] or 100 doses of a nebulized rescue medication); and (4) count of baseline maintenance units dispensed ( 1 unit of maintenance medication was defined as a 30-day supply). Medication units were determined using pharmacy claims. Further information on the classification of patients by baseline exacerbation history is in the online data supplement.

Clinical characteristics examined at baseline included: COPD medication use, COPD exacerbations, type of COPD diagnosis, and procedures (Table 1).

\section{Outcome}

The primary outcome was the occurrence of severe exacerbations during the 1-year at-risk period. The definition of a severe exacerbation during the atrisk period was identical to the definition used in the baseline period, with the addition of the corresponding ICD-10 codes: J41-J44, for an inpatient hospital stay with a diagnosis of COPD as a primary diagnosis; or J80, J9600-J9602, or J9620-J9622 as a secondary diagnosis with a primary diagnosis of respiratory failure. Exacerbations beginning during the baseline period and continuing into the at-risk period were attributed to the baseline period.

\section{Statistical Analysis}

Baseline demographics and clinical characteristics were described overall and stratified by severe exacerbation occurrence during the at-risk period. Unadjusted and adjusted logistic regression models were estimated for the association between the outcome of severe exacerbation versus no exacerbation and the 4 predictors of interest. Variables included in the adjusted model were age, geographic region in which the person was enrolled, ${ }^{15}$ chronic oxygen, and nebulizer use. The $C$-statistic was used to compare the predictive ability of each of the predictors. The odds ratio (OR) with 95\% confidence interval (CI), $P$-value, and $C$-statistic were calculated for each model. The a priori alpha level for all inferential analyses was set at 0.05 , and all statistical tests were 2 -tailed. All statistical analyses were performed using SAS 9.4 software.

\section{Results}

\section{Study Population}

In total, 60,776 MAPD beneficiaries were identified for inclusion in this analysis; 49,133 had no exacerbation and $11,643 \mathrm{had} \geq 1$ severe exacerbation during the atrisk period (Figure 2).

\section{Descriptive Analysis}

The study population had a mean age ( \pm standard deviation) of $71.0( \pm 9.1)$ years and was predominantly female (58.6\%; Table 1). Compared with patients who experienced no exacerbation, those who experienced $\geq 1$ severe exacerbation during the at-risk period used significantly more COPD medications (including frequent $[\geq 4$ fills $]$ systemic corticosteroids use $[22.9 \%$ versus $6.7 \%]$, use of $\geq 2$ maintenance medication classes $[77.9 \%$ versus $70.1 \%]$, and frequent $[\geq 4$ units] rescue medication use [55.0\% versus $32.5 \%]$ ), experienced significantly more baseline COPD exacerbations ( $\geq 2$ severe exacerbations: $14.2 \%$ versus $1.6 \%$, respectively; $P<0.001$ ), and had more procedures (chronic oxygen therapy and nebulizer) during the baseline period (Table 1). Individuals with a CTR of $<.1$ during baseline were most likely to experience an exacerbation during the at-risk period, while those with a CTR of 1 in the baseline period were least likely to experience an exacerbation during the at-risk period (Figure 3).

\section{Outcome Measures}

In the unadjusted analyses, the best predictor of severe exacerbation during the at-risk period, was the logistic regression model that included any baseline exacerbation, which demonstrated the highest C-statistic of unadjusted models (0.668); this was followed by the number of rescue units dispensed (0.651), CTR (0.619), and the number of maintenance units dispensed (0.562) (Table 2). Any baseline exacerbation, a higher number of rescue units and higher number of maintenance units, were positively associated with having $\geq 1$ severe exacerbation (an OR $>$ 1.0) during the at-risk period. Conversely, baseline CTR was inversely associated $(\mathrm{OR}<1.0)$ with having $\geq$ 1 severe exacerbation during the at-risk period. 


\section{Table 1. Baseline Risk Factors for Severe COPD Exacerbation Overall and Stratified by Exacerbation Status During the At-Risk Period}

\begin{tabular}{|c|c|c|c|}
\hline Baseline Variables ${ }^{a}$ & $\begin{array}{c}\text { Total } \\
(\mathrm{N}=60,776)\end{array}$ & $\begin{array}{l}\text { No Exacerbation(s) } \\
\qquad(n=49,133)\end{array}$ & $\begin{array}{l}\geq 1 \text { Severe Exacerbation(s) } \\
\qquad(n=11,643)\end{array}$ \\
\hline Female, n (\%) & $35,602(58.6)$ & $28,504(58.0)$ & $7098(61.0)$ \\
\hline Age, mean (SD), years & $71.0(9.1)$ & $71.0(9.1)$ & $70.7(9.2)$ \\
\hline \multicolumn{4}{|l|}{ COPD Medication, $\mathrm{n}(\%)$} \\
\hline \multicolumn{4}{|l|}{ Systemic Corticosteroids } \\
\hline$\geq 1$ fill & $26,145(43.0)$ & $18,744(38.2)$ & 7401 (63.6) \\
\hline$\geq 4$ fill & $5937(9.8)$ & $3271(6.7)$ & $2666(22.9)$ \\
\hline \multicolumn{4}{|l|}{ Maintenance Classes ${ }^{b}$} \\
\hline 1 & $17,247(28.4)$ & $14,677(29.9)$ & $2570(22.1)$ \\
\hline$\geq 2$ & $43,529(71.6)$ & $34,456(70.1)$ & $9073(77.9)$ \\
\hline \multicolumn{4}{|l|}{ Canisters of Rescue Medication } \\
\hline$\geq 1$ fill & $40,738(67.0)$ & $31,272(63.7)$ & $9466(81.3)$ \\
\hline$\geq 4$ units & $22,385(36.8)$ & $15,983(32.5)$ & $6402(55.0)$ \\
\hline \multicolumn{4}{|c|}{ COPD Exacerbations, $n(\%)$} \\
\hline \multicolumn{4}{|c|}{ Count of Any Moderate or Severe Exacerbation Categories } \\
\hline 0 & $41,258(67.9)$ & $36,518(74.3)$ & $4740(40.7)$ \\
\hline 1 & $12,426(20.5)$ & $9310(19.0)$ & $3116(26.8)$ \\
\hline$\geq 2$ & $7092(11.7)$ & $3305(6.7)$ & $3787(32.5)$ \\
\hline \multicolumn{4}{|c|}{ Count of Severe Exacerbation Categories } \\
\hline 0 & $51,310(84.4)$ & $44,045(89.6)$ & 7265 (62.4) \\
\hline 1 & $7040(11.6)$ & $4317(8.8)$ & $2723(23.4)$ \\
\hline$\geq 2$ & $2426(4.0)$ & $771(1.6)$ & $1655(14.2)$ \\
\hline \multicolumn{4}{|l|}{ Type of COPD Diagnosis, ${ }^{\mathbf{c}} \mathrm{n}(\%)$} \\
\hline Chronic Bronchitis & $24,653(40.6)$ & $17,194(35.0)$ & $7459(64.1)$ \\
\hline Emphysema & $10,908(18.0)$ & $7596(15.5)$ & $3312(28.5)$ \\
\hline Chronic Airway Obstruction & $51,773(85.2)$ & $40,763(83.0)$ & $11,010(94.6)$ \\
\hline \multicolumn{4}{|l|}{ Procedures, $\mathrm{n}(\%)$} \\
\hline Chronic Oxygen Therapy & $17,905(29.5)$ & $11,789(24.0)$ & $6116(52.5)$ \\
\hline Nebulizer & $20,543(33.8)$ & $14,448(29.4)$ & $6095(52.4)$ \\
\hline COPD Treatment Ratio, mean (SD) & $0.71(0.3)$ & $0.73(0.3)$ & $0.62(0.3)$ \\
\hline
\end{tabular}

All differences between the 'No Exacerbation' and 'Severe Exacerbation' groups were statistically significant; $P<0.001$.

${ }^{a}$ Baseline period is defined as the first year following the index date.

${ }^{b}$ Maintenance class count was based on the following maintenance medication classes: long-acting antimuscarinic agents, long-acting

beta2-agonists, inhaled corticosteroids, methylxanthines, and phosphodiesterase-4 enzyme inhibitor.

${ }^{\mathrm{C}} \mathrm{COPD}$ diagnoses are based on $\geq 1$ claim for specific diagnosis type during the baseline period and are not mutually exclusive.

$\mathrm{COPD}=$ chronic obstructive pulmonary disease; $\mathrm{SD}=$ standard deviation

The patterns were similar, but with increased $C$-statistic values, after adjustment for age, geographic region, chronic oxygen, and nebulizer use, explained below. The adjusted $\mathrm{OR}$ of a severe exacerbation was 0.90 (95\% CI, 0.89-0.91) per 0.10 change in CTR (C-statistic, $0.710 ; P<0.001)$. When any baseline exacerbation was the predictor of interest, the adjusted OR of a severe exacerbation was 3.02 (95\% CI, 2.89-
3.16; $C$-statistic, 0.734; $P<0.001)$. The adjustment with inclusion of both any baseline exacerbation and CTR and all previously mentioned predictors resulted in a $C$-statistic of 0.742 , and the $O R$ of a severe exacerbation was 0.92 (95\% CI, 0.91-0.93) per 0.10 change in CTR $(P<0.001)$. 


\section{Figure 2. Sample Selection}

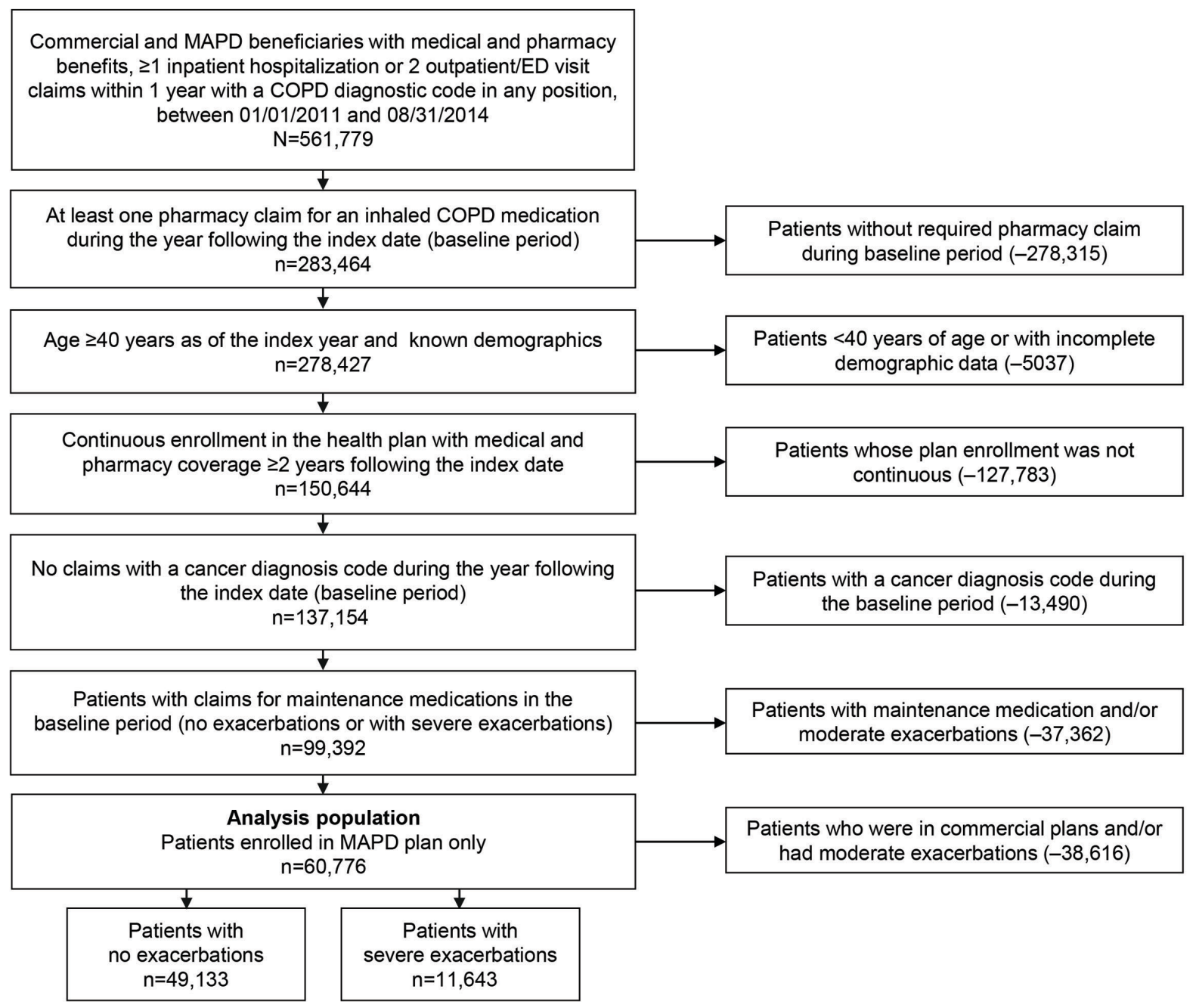

$\mathrm{MAPD}=$ Medicare Advantage with Part D coverage; $\mathrm{ED}=$ emergency department; $\mathrm{COPD}=$ chronic obstructive pulmonary disease

\section{Discussion}

This retrospective observational study utilized U.S. administrative claims data to validate and compare the CTR, a modifiable measure of COPD exacerbation risk, to other COPD exacerbation predictors in a group of MAPD beneficiaries diagnosed with COPD. The study found that, while previous exacerbation was the strongest predictor for future exacerbation, the CTR was an effective measure for identification of people diagnosed with COPD who are at increased risk of severe exacerbation.

As expected, the mean baseline CTR was lower (i.e., higher rescue medication use relative to maintenance use) among those who experienced a severe exacerbation during the at-risk period, compared with individuals who experienced no exacerbation during the at-risk period (data not shown). The $C$-statistic from the unadjusted and adjusted models was highest for any baseline exacerbation, corroborating previous studies that found prior exacerbation to be the strongest predictor for a future exacerbation. ${ }^{16,17}$ In the original CTR validation study, ${ }^{13}$ the CTR performed well in predicting exacerbation risk at a population level using claims data, reporting $C$-statistics between 0.714 and 0.761 , which compares favorably with other risk models that have utilized clinical information. ${ }^{9-12,18}$

Previous analyses have included populations that 
Figure 3. Percentage of Patients Diagnosed with COPD who Experienced a Severe Exacerbation During the At-Risk Period by Baseline COPD Treatment Ratio

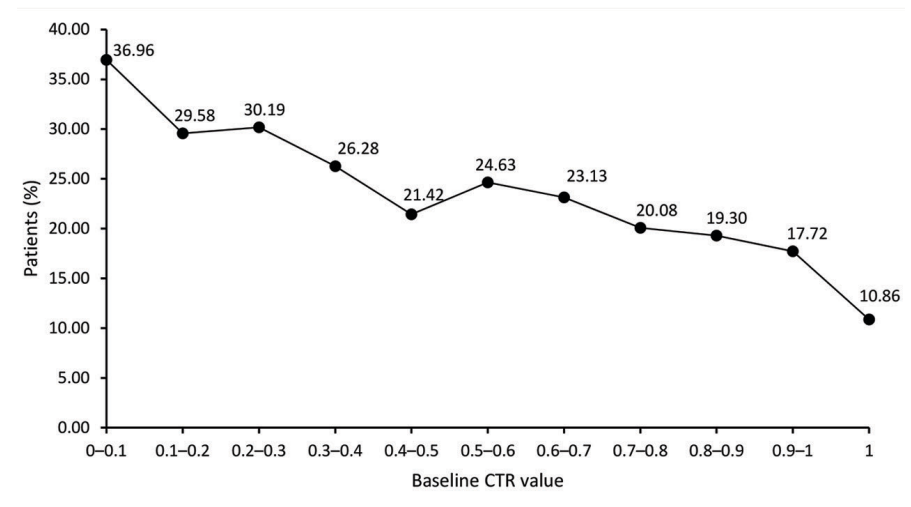

Note: all data points, except $\mathrm{CTR}=1$, are the average across the CTR range, as indicated on the $\mathrm{x}$-axis. The lower bound of each range is excluded and the lower and upper bound are excluded from the 0.9-1.0 range.

$\mathrm{COPD}=$ chronic obstructive pulmonary disease; $\mathrm{CTR}=\mathrm{COPD}$ treatment ratio comprise a mix of commercial and Medicare patients. ${ }^{13}$ The final analysis in this study was limited to those with MAPD coverage with maintenance medication, to further assess the predictive ability of the CTR in the Medicare population.

More recently, several models for predicting exacerbation risk were examined to help identify variables that are important in predicting severe COPD exacerbation risk, ${ }^{19}$ although no model demonstrated reliable predictors among data available in medical claims. Other studies have examined clinical and biomarker data, ${ }^{20}$ but results could not be replicated in additional samples. The asthma medication ratio (AMR) is an example of a practical measure for use in community practice to help enhance quality of practice and reduce morbidity, mortality, and health care costs. $^{21,22}$ The AMR allows health care organizations to use pharmacy data to identify and target people diagnosed with asthma who require intervention to subsequently enhance outcomes (National Committee for Quality Assurance ${ }^{23}$ ), and is a beneficial tool for payers, decision makers, and physician groups who want to identify gaps in exacerbation prevention and medical adherence. The CTR is calculated in a similar manner to the AMR and could easily be incorporated

\section{Table 2. Unadjusted and Adjusted Association of Selected Risk Factors and Severe Exacerbation(s) During the At-Risk Period}

\begin{tabular}{|c|c|c|c|}
\hline Covariate(s) of Interest ${ }^{a}$ & Adjusters $^{a}$ & C-statistic & OR $(95 \% \mathrm{Cl})^{\mathrm{b}}$ \\
\hline $\mathrm{CTR}^{\mathrm{C}}$ & & 0.619 & $0.865(0.858-0.871)$ \\
\hline Any Baseline Exacerbation $(\mathrm{y} / \mathrm{n})$ & & 0.668 & $4.216(4.042-4.397)$ \\
\hline $\begin{array}{l}\text { Number of Rescue Units } \\
\text { Dispensed }^{\mathrm{d}}\end{array}$ & & 0.651 & $1.062(1.059-1.065)$ \\
\hline \multirow[t]{2}{*}{$\begin{array}{l}\text { Number of Maintenance Units } \\
\text { Dispensed }^{\mathrm{d}}\end{array}$} & & 0.562 & $1.032(1.029-1.034)$ \\
\hline & Chronic Oxygen, Nebulizer Use, Age, Geographic Region & 0.693 & $0.900(0.893-0.907)$ \\
\hline CTR $^{\mathrm{C}}$ & Chronic Oxygen, Nebulizer Use, Age, Geographic Region & 0.710 & $3.024(2.890-3.164)$ \\
\hline Any Baseline Exacerbation $(\mathrm{y} / \mathrm{n})$ & Chronic Oxygen, Nebulizer Use, Age, Geographic Region & 0.734 & $1.045(1.042-1.048)$ \\
\hline $\begin{array}{l}\text { Number of Rescue Units } \\
\text { Dispensed }^{\mathrm{d}}\end{array}$ & Chronic Oxygen, Nebulizer Use, Age, Geographic Region & 0.718 & $1.021(1.019-1.024)$ \\
\hline $\begin{array}{l}\text { Number of Maintenance Units } \\
\text { Dispensed }^{\mathrm{d}}\end{array}$ & Chronic Oxygen, Nebulizer Use, Age, Geographic Region & 0.700 & $0.919(0.911-0.926)$ \\
\hline $\begin{array}{l}\text { CTR }^{C} \\
\text { Any Baseline Exacerbation }\end{array}$ & Chronic Oxygen, Nebulizer Use, Age, Geographic Region & 0.742 & $2.856(2.728-2.989)$ \\
\hline
\end{tabular}

aBlank cells for covariate of interest or adjusters indicate that there were no variables of that type in the model;

${ }^{b}$ All odds ratios were statistically significant, $p<0.001$;

continuous, 0.1-unit increment;

$\mathrm{d}_{\text {continuous, } 1 \text {-unit increment; }}$

$\mathrm{OR}=$ odds ratio; $\mathrm{CI}=$ confidence interval $; \mathrm{CTR}=\mathrm{COPD}$ treatment ratio 
as a quality measure alongside the asthma measure for patients diagnosed with COPD.

Compared with other tools for predicting future exacerbation in people diagnosed with COPD, the CTR offers several advantages. Firstly, it utilizes pharmacy claims data, which are readily accessible and can therefore be calculated when other forms of data are unavailable. For example, while prior exacerbation history is the strongest predictor of future exacerbation and many clinical variables may contribute to that risk, these types of data are not always available, especially to national quality-of-care organizations. Secondly, the CTR is a modifiable risk factor. This means that individuals with a low ratio of maintenance medication to rescue medication can be identified and, with appropriate medical intervention and adherence support, can increase the ratio of maintenance to rescue medication. This would allow a program to be developed with the goal of modifying the ratio of maintenance to rescue medication, compared with a program solely focused on the reduction of rescue medication. Finally, the CTR can be used as a valuable guide for organizations making decisions about whether to intervene and dedicate services to exacerbation prevention, specifically allowing those with fewer resources to choose a lower score (e.g., $\leq 0.2$ ) as a cut-off point for identification of high-risk patients, and vice versa.

This study has several limitations that must be considered when interpreting its findings. Exacerbation events were identified according to a definitional algorithm using health insurance claims data; the use of an algorithm with less than 100\% sensitivity and specificity will result in misclassification of the outcome. All variables used in the analysis were defined based on the presence of codes on administrative claims, which does not guarantee that the beneficiary received that diagnosis, underwent a procedure, or received a specific medication. While candidate covariates for multivariable analysis in this study were limited to health insurance claims data, disregarding important clinical measures in COPD (e.g., lung function or oxygen saturation), they reflected those used to develop the CTR. ${ }^{13}$ However, prior research found similar predictive accuracy of the CTR models with and without the Global initiative for chronic Obstructive Lung Disease stage. ${ }^{13}$ Finally, the results of this analysis are primarily applicable to COPD patients in stable managed care settings and may not be generalizable to the U.S. COPD population as a whole.

\section{Conclusions}

This study found that the CTR is an effective tool for identifying people diagnosed with COPD who are at increased risk for severe exacerbation. While the CTR does not predict severe exacerbation as well as the presence of a prior exacerbation, the CTR can measure the risk of exacerbation in patients without a history of exacerbations, and can be assessed without the need for medical history; in addition, it can be used to modify patient management appropriately, particularly in low resource settings. Overall, the CTR is an effective tool for predicting severe exacerbation risk and performed similarly to other pharmacy-based predictors explored in this study. It is suggested that future investigation focuses on determining its predictive value across differing lengths of baseline and at-risk periods, testing the CTR against other pharmacy-based predictors.

\section{Acknowledgments}

Study design consultation was provided by Ami Buikema; programming support by Yiyu Fang; and project management by Sharanya Murali and Caroline Jennermann, all of Optum. Editorial support (in the form of writing assistance, collating author comments, assembling tables/figures, grammatical editing and referencing) was provided by Tom Gallagher, $\mathrm{PhD}$, of Gardiner-Caldwell Communications (Macclesfield, UK) and was funded by GlaxoSmithKline plc.

Author Contributions: All authors performed data analysis and/or data interpretation. RHS and LGSB conceived and/or designed the study; SK and LGSB contributed to the acquisition of data; and all authors revised the report for important intellectual content and approved the final article for submission.

Data availability: The data contained in our database contains proprietary elements owned by Optum and, therefore, cannot be broadly disclosed or made publicly available at this time. The disclosure of this data to third-party clients assumes certain data security and privacy protocols are in place, and that the third-party client has executed our standard license agreement which includes restrictive covenants governing the 
use of the data.

\section{Declaration of Interest}

The authors declare the following conflicts of interest during the last 3 years in relation to this article: At the time of the study, RHS was an employee of GlaxoSmithKline plc and holds stock options/shares. At the time of the study, TR was a fellow at UNC. SK, LB, and LGSB are employees of Optum; GlaxoSmithKline plc contracted with Optum to conduct this research. 


\section{References}

1. Global Initiative for Chronic Obstructive Lung Disease (GOLD). Global strategy for the diagnosis, management, and prevention of chronic obstructive pulmonary disease; 2019 report. GOLD website. https://goldcopd.org/wp-content/uploads/2018/11/ GOLD-2019-v1.7-FINAL-14Nov2018-WMS.pdf.

Published 2018. Accessed November 1, 2018.

2. Croft JB, Wheaton AG, Liu Y, et al. Urban-rural county and state differences in chronic obstructive pulmonary disease - United States, 2015. MMWR. Morb Mortal Wkly Rep. 2018;67(7):205211. doi: https://doi.org/10.15585/mmwr.mm6707a1

3. Ford ES, Murphy LB, Khavjou O, et al. Total and state-specific medical and absenteeism costs of COPD among adults aged $\geq 18$ years in the United States for 2010 and projections through 2020. Chest. 2015;147(1):31-45.

doi: https://doi.org/10.1378/chest.14-0972

4. Balcells E, Antó JM, Gea J, et al. Characteristics of patients admitted for the first time for COPD exacerbation. Respir Med. 2009; 103(9):1293-1302.

doi: https://doi.org/10.1016/j.rmed.2009.04.001

5. Bartles W, Adamson S, Leung L, Sin DD, van Eeden SF. Emergency department management of acute exacerbations of chronic obstructive pulmonary disease: factors predicting readmission. Int J Chron Obstruct Pulmon Dis. 2018;13:16471654. doi: https://doi.org/10.2147/COPD.S163250

6. Blanchette CM, Dalal AA, Mapel D. Changes in COPD demographics and costs over 20 years. J Med Econ. 2012;15(6):1176-1182.

doi: https://doi.org/10.3111/13696998.2012.713880

7. Blanchette CM, Gross NJ, Altman P. Rising costs of COPD and the potential for maintenance therapy to slow the trend. Am Health Drug Benefits. 2014;7(2):98-106.

8. Mapel DW, Schum M, Lydick E, Marton JP. A new method for examining the cost savings of reducing COPD exacerbations. Pharmacoeconomics. 2010;28(9):733-749. doi: https://doi.org/10.2165/11535600-000000000-00000

9. Niewoehner DE, Lokhnygina $\mathrm{Y}$, Rice $\mathrm{K}$, et al. Risk indexes for exacerbations and hospitalizations due to COPD. Chest. 2007;131(1):20-28. doi: https://doi.org/10.1378/chest.06-1316

10. Marin JM, Carrizo SJ, Casanova C, etal. Prediction of risk of COPD exacerbations by the BODE index. Respir Med. 2009;103(3):373378. doi: https://doi.org/10.1016/j.rmed.2008.10.004

11. Bertens LC, Reitsma JB, Moons KG, et al. Development and validation of a model to predict the risk of exacerbations in chronic obstructive pulmonary disease. Int $J$ Chron Obstruct Pulmon Dis. 2013;8:493-499.

doi: https://doi.org/10.2147/COPD.S49609
12. Lee SD, Huang MS, Kang J, et al; Investigators of the Predictive Ability of CAT in Acute Exacerbations of COPD (PACE) study. The COPD Assessment Test (CAT) assists prediction of COPD exacerbations in high-risk patients. Respir Med. 2014;108(4):600608. doi: https://doi.org/10.1016/j.rmed.2013.12.014

13. Stanford RH, Lau MS, Li Y, Stemkowski S. External validation of a COPD risk measure in a commercial and Medicare population. J Manag Care Spec Pharm. 2019;25(1):58-69. doi: https://doi.org/10.18553/jmcp.2019.25.1.058

14. Stanford RH, Nag A, Mapel DW, et al. Claims-based risk model for first severe COPD exacerbation. Am J Manag Care. 2018;24(2):e45-e53.

15. United States Census Bureau. Census regions and divisions of the United States. U.S. Census website. http://www2.census.gov/ geo/pdfs/maps-data/maps/reference/us_regdiv.pdf.

Published 2015. Accessed November 1, 2018.

16. Hurst JR, Vestbo J, Anzueto A, et al. Susceptibility to exacerbation in chronic obstructive pulmonary disease. $N$ Engl J Med. 2010;363(12):1128-1138.

doi: https://doi.org/10.1056/NEJMoa0909883

17. Jenkins CR, Celli B, Anderson JA, et al. Seasonality and determinants of moderate and severe COPD exacerbations in the TORCH study. Eur Respir J. 2012;39(1):38-45. doi: https://doi.org/10.1183/09031936.00194610

18. Miravitlles M, Guerrero T, Mayordomo C, Sánchez-Agudo L, Nicolau F, Segú JL. Factors associated with increased risk of exacerbation and hospital admission in a cohort of ambulatory COPD patients: a multiple logistic regression analysis. Respiration. 2000;67(5):495-501. doi: https://doi.org/10.1159/000067462

19. Samp JC, Joo MJ, Schumock GT, Calip GS, Pickard AS, Lee TA. Predicting acute exacerbations in chronic obstructive pulmonary disease. J Manag Care Spec Pharm. 2018;24(3):265279. doi: https://doi.org/10.18553/jmcp.2018.24.3.265

20. Keene JD, Jacobson S, Kechris K, et al. Biomarkers predictive of exacerbations in the SPIROMICS and COPDGene cohorts. Am J Respir Crit Care Med. 2017;195(4):473-481. doi: https://doi.org/10.1164/rccm.201607-13300C

21. Schatz M, Nakahiro R, Crawford W, Mendoza G, Mosen D, Stibolt TB. Asthma quality-of-care markers using administrative data. Chest. 2005;128:1968-1973. doi: https://doi.org/10.1378/chest.128.4.1968

22. Broder MS, Gutierrez B, Chang E, Meddis D, Schatz M. Ratio of controller to total asthma medications: determinants of the measure. Am J Manag Care. 2010;16(3):170-178. 
23. National Committee for Quality Assurance (NCQA). Medication management for people with asthma and asthma medication ratio (MMA, AMR). NCQA website. https://www.ncqa.org/ hedis/measures/medication-management-for-people-withasthma-and-asthma-medication-ratio/.

Published 2018. Accessed November 1, 2018. 